Rev. Adm. Saúde (On-line), São Paulo, v. 21, n. 82: e277, jan. - mar. 2021, Epub 31 mar. 2021 http://dx.doi.org/10.23973/ras.82.277

ARTIGO ORIGINAL

\title{
Aplicação de análise descritiva para compreensão das atividades de desospitalização de um hospital universitário de grande porte entre 2009 e 2019
}

Application of descriptive analytics to understand the de-hospitalization activities of a large university hospital between 2009 and 2019

\section{Antonio Valerio Netto ${ }^{1}$, Aline Carvalho ${ }^{2}$, Luciano Rodrigues de Oliveira ${ }^{3}$}

1. Bacharel em ciência da computação. Professor visitante em health data science e telemedicina na Escola Paulista de Medicina da Universidade Federal de São Paulo (UNIFESP), São Paulo SP

2. UNIFESP, São Paulo SP

3. Enfermeiro. Doutorando em ciências da saúde pela UNIFESP, São Paulo SP

\section{RESUMO}

A análise descritiva (descriptive analytics) permite responder perguntas relacionadas ao que aconteceu durante um determinado período. Ao desenvolver indicadores chaves de desempenho, a análise das informações pode ajudar a rastrear atividades de sucessos ou fracassos. É possível gerar métricas especializadas para acompanhar o desempenho em setores específicos, como é o caso do ambiente hospitalar, ou mesmo identificar métricas necessárias para a análise e a melhoria da gestão. Esse processo requer coleta, processamento, análise e visualização de dados relevantes. Ele fornece uma visão essencial do desempenho no passado. Diante disso, a proposta do artigo está relacionada à aplicação da análise descritiva para identificar as atividades de desospitalização em um hospital universitário de grande porte da cidade de São Paulo (SP). O artigo também discorre sobre os principais conceitos correlatos entre a transição de cuidado e os indicadores de 
DRG (Diagnosis Related Groups), principalmente, taxa de reinternação hospitalar e eficiência do uso do leito. Baseada nessas análises busca-se influenciar os gestores hospitalares para que possam refletir e promover uma melhoria contínua nos processos relacionados à jornada do paciente.

Palavras-chave: Estratégias de Saúde, Gestão Hospitalar, Cuidado de Transição, Assistência ao Paciente, Relatórios Públicos de Dados de Saúde.

\begin{abstract}
Descriptive analytics allows you to answer questions related to what happened during a certain period. In developing key performance indicators, analyzing information can help track success or failure activities. It is possible to generate specialized metrics to monitor performance in specific sectors, such as the hospital environment, or even to identify metrics necessary for the analysis and improvement of management. This process requires the collection, processing, analysis, and visualization of relevant data. It provides an essential view of past performance. Therefore, the proposal of the article is related to the application of descriptive analysis to identify the activities of rehospitalization in a large university hospital in the city of São Paulo (SP). The article also discusses the main correlated concepts between the transition of care and the indicators of DRG (Diagnosis Related Groups), mainly, rate of hospital readmission and efficiency in the use of the bed. Based on these analyzes, we seek to influence hospital managers so that they can reflect and promote continuous improvement in processes related to the patient's journey.
\end{abstract}

Keywords: eHealth Strategies, Hospital Organization and Administration, Transition Care, Patient Care, Public Reporting of Healthcare Data.

\title{
INTRODUÇÃO
}

O processo de melhoria contínua da gestão hospitalar passa pela análise de seu conjunto de dados internos para que seja possível tecer considerações para promover modificações em seu planejamento e execução de atividades. Inclusive, em 1991, reconhecendo a importância da gestão dos dados hospitalares foi criado o departamento de informática do Sistema Único de Saúde (DATASUS), com a criação da Fundação Nacional de Saúde (Funasa). $\mathrm{Na}$ época, a fundação passou a exercer a função de controle e processamento das contas referentes à saúde que antes era da Empresa de Tecnologia e Informações da Previdência Social (DATAPREV). O DATASUS tem como responsabilidade prover sistemas de informação e suporte de informática, necessários ao processo de planejamento, operação e controle ${ }^{1}$. 
Para gerenciar as informações das bases de dados foi desenvolvido o TABNET. Trata-se de um tabulador genérico de domínio público que permite organizar dados de forma rápida conforme a consulta que se deseja tabular ${ }^{2}$. Por meio dele, o DATASUS disponibiliza informações que podem servir para subsidiar análises objetivas da situação sanitária e tomadas de decisão baseadas em elaboração de programas de ações de saúde. São informações sobre assistência à saúde da população, cadastros (rede assistencial), redes hospitalares e ambulatoriais, cadastro dos estabelecimentos de saúde, além de informações sobre recursos financeiros e informações demográficas e socioeconômicas ${ }^{3}$.

As fontes de dados do DATASUS são classificadas em quatro grupos: sistemas de informações do Ministério da Saúde, outros sistemas de informações de entidades públicas, censos e pesquisas provenientes do Sistema Estatístico Nacional, operados pelo Instituto Brasileiro de Geografia e Estatística (IBGE) e outros censos e pesquisas. O Sistema de Informações Hospitalares do SUS (SIH/SUS) foi originalmente concebido como Sistema de Assistência MédicoHospitalar da Previdência Social (SAMHPS) para operar o sistema de pagamento de internação aos hospitais contratados pelo Ministério da Previdência Social, a partir de 1981. A partir da constituição federal de 1988, o SAMHPS foi estendido em 1991 para todo o SUS, incluindo os hospitais públicos municipais, estaduais e federais (administração indireta e outros ministérios), transformando-se no SIH/SUS.

Atualmente, o SIH/SUS é gerido pela Secretaria de Assistência à Saúde, sendo seu documento básico a "Autorização de Internação Hospitalar" (AlH), que habilita a internação do paciente e gera valores para pagamento. A AlH é preenchida pelo estabelecimento hospitalar e enviada mensalmente, em meio magnético, ao gestor municipal e/ou estadual do SUS, conforme o nível de gestão, para consolidação junto ao DATASUS. São disponibilizados dados individualizados (não identificados) sobre o paciente e a internação, como também o diagnóstico de internação, os procedimentos realizados, os valores pagos, entre outras informações. A abrangência do sistema está limitada às internações no âmbito do SUS. Estima-se que o SIH/SUS reúna informações sobre 60 a 70\% das internações hospitalares realizadas no país, variando de acordo com a região. Contudo, eventuais reinternações e transferências do mesmo paciente a outros hospitais também não são identificadas, o que pode resultar em contagem cumulativa 4 .

Diante desse conjunto de dados, faz-se necessário a aplicação de algoritmos analíticos para identificar possíveis padrões, e posteriormente, construir uma base histórica de dados de gestão hospitalar que podem e devem fazer uso de plataformas de $\mathrm{BI}$ (Business Intelligence) / BA (Business Analytics) ou mesmo Big Data para construir um processo de análise de informações e tomada de decisão. Neste caso, se faz uso do Health Analytics. Trata-se de um conjunto de metodologias utilizadas para analisar grandes volumes de dados relacionados com o setor de saúde, contribuindo no cuidado clínico geral e na gestão dos recursos de saúde. Para os gestores de saúde, essa contribuição está em reduzir desperdícios e melhorar a qualidade e a produtividade do cuidado com a saúde dos beneficiários, sempre de forma sustentável. Tanto Health Data Science ${ }^{5}$ quanto Health Analytics fazem uso das aplicações em 
Data Analytics cujo objetivo é examinar dados brutos para encontrar padrões e tirar conclusões sobre essa informação, aplicando um processo algorítmico ou mecânico para obter informações. Data Analytics é um campo amplo e existem quatro tipos principais de análise de dados: Descriptive analytics, Diagnostic analytics, Predictive analytics e Prescriptive analytics ${ }^{6}$.

No caso do analítico descritivo, o interesse é saber o que aconteceu durante um determinado período ${ }^{7}$. Sobre $o$ analítico diagnóstico, o interesse é saber 0 porquê aconteceu. Isto é, se aprofundar nos dados coletados dos pacientes, a fim de entender "Por que algumas coisas aconteceram". Para isto, é possível usar ferramentas de $\mathrm{Bl}$ para obter alguns insights. No entanto, é um trabalho penoso e que tem capacidade limitada. Basicamente, este tipo de algoritmo fornece um bom entendimento de uma parte limitada do problema que se pretende resolver. Para o analítico preditivo o foco é compreender o que pode acontecer. Se for possível coletar dados contextuais e correlacioná-los com outros conjuntos de dados de comportamento do paciente, por exemplo, será possível prever o que vai acontecer se for mantida a situação atual. Por fim, o uso do analítico prescritivo busca entender como prevenir as situações ruins e potencializar as boas. Assim que chegar ao ponto da análise de dados consistentemente prever o que vai acontecer, é possível ser capaz de entender o que deve ser realizado a fim de maximizar os bons resultados e também minimizar os resultados potencialmente ruins. Importante comentar que para ser capaz de implementar os analíticos preditivos e prescritivos é necessário adicionar uma tomada de decisão automática a esta análise por meio de algoritmos de aprendizado de máquina (machine learning), como por exemplo, redes neurais, lógica fuzzy, SVM (support vector machine), entre outros.

\section{Longa permanência hospitalar e desospitalização}

O aumento do tempo de permanência hospitalar - um dos pontos que refletem a mudança da pirâmide etária e do perfil epidemiológico do país - é um entrave, afetando a saúde financeira dos hospitais. A longa permanência, isto é, quando o tempo de internação é maior ou igual a 30 dias; aumenta os custos e reduz a oportunidade de outros pacientes em estágio agudo da doença receberem atenção hospitalar, reduzindo assim a rotatividade dos leitos ${ }^{8}$. Esse aumento de custos iniciado no hospital é redirecionado para o SUS, operadoras de saúde e, na sequência, para suas principais financiadoras: as empresas. Somente a Anahp (Associação Nacional de Hospitais Privados) afirma que, em 2017, mais de 9.170 pacientes ficaram por mais de 90 dias em longa permanência em seus hospitais 9 .

A saúde é uma das vertentes econômicas que precisam de maior maleabilidade para se adequar aos diferentes cenários que se desenham dentro de uma grande economia como a brasileira. As mudanças de perfis epidemiológicos, somadas à crescente demanda por economia de recursos em uma nação que vem enfrentando crises políticas e econômicas recorrentes, exigem que o cenário de saúde seja constantemente adaptado para garantir um atendimento de qualidade e, acima de tudo, seguro para todos os cidadãos. Importante que essa análise de dados seja realizada de forma contínua e em 
tempo hábil para que as adequações administrativas sejam executadas o quanto antes, minimizando problemas futuros.

Atualmente, existem alternativas viáveis para a liberação dos leitos dos hospitais de alta complexidade. Muitas vezes, esses pacientes internados por longos períodos poderiam estar sendo atendidos em outras unidades de saúde de menor complexidade. E consequentemente com menor custo de manutenção, recebendo tratamento adequado, além de menor exposição a possíveis infecções; onerando menos o sistema. Já existem resultados consolidados em diversos locais do mundo que utilizam os serviços de desospitalização conhecidos internacionalmente como Post Acute Care (Cuidado Pós-Agudo) ${ }^{10}$.

No Brasil, estes serviços foram criados baseados nos modelos internacionais com adaptações para atender questões locais. Um exemplo são as unidades de retaguarda, cuidados paliativos e transição que ganham força no país. São locais onde esses pacientes que não mais precisam dos cuidados intensivos de um hospital, mas ainda não podem retomar suas atividades normais (carecem de cuidados específicos) encontram uma infraestrutura completa e adaptada às suas necessidades. Ao mesmo tempo, os planos de saúde encontram uma possibilidade de manter um atendimento de qualidade reduzindo de forma significativa seus custos ${ }^{11}$. O maior problema enfrentado com relação a falta de leitos de retaguarda é o uso de leitos de alta complexidade com pacientes que já poderiam estar sendo cuidados em locais com menos recursos tecnológicos e custos de manutenção.

\section{METODOLOGIA}

Foi realizada uma revisão de literatura de caráter descritivo, com abordagem quantitativa, retrospectiva e crítica com uma análise de dados utilizando as bases do departamento de informática em saúde do Sistema Único de Saúde (DATASUS). Aplicou-se uma análise descritiva dos dados utilizando a ferramenta TT Hospital Explorer da empresa Techtrials em um hospital universitário de grande porte da cidade de São Paulo (SP). A ferramenta utiliza dados coletados periodicamente das bases do Sistema de Informações Ambulatoriais de Saúde (SIA/SUS) e do Sistema de Informações Hospitalares (SIH/SUS), além do Cadastro Nacional de Estabelecimentos de Saúde (CNES). Os gráficos e os dados utilizados foram planejados buscando informações das entradas de pacientes, reinternações, diagnósticos de entrada e saída, tipo de saída, transição de cuidado, perfil dos pacientes, entre outros.

Foi utilizado para análise, o banco de dados disponibilizado pelo DATASUS entre os anos de 2009 e 2019. Para estudar os dados de reinternação foi criado um business key composto por três campos. São eles: CEP (Código de Endereçamento Postal), data de nascimento e sexo. Esse business key foi validada por um estudo de caso envolvendo 7.367 hospitalizações de cirurgia bariátrica (banco de dados fornecido pelo DATASUS). Foram cruzados os dados das AlHs com o CNS (Cartão Nacional de Saúde) de cada paciente; e 
identificadas todas as internações e reinternações. Para o mesmo conjunto de dados, foi aplicada a business key criada e a taxa de acerto foi de $90,55 \%$.

Foram empregados dois indicadores para análise da desospitalização: altas para assistência domiciliar ou transferências para instituições de longa permanência ou serviços ambulatoriais específicos; e o número de pacientes com tempo de permanência superior a 30 dias desospitalizados para estes serviços. Referente a taxa de reinternação, foram levantados o número de pacientes com reinternação em menos de 30 dias e os que apresentaram o mesmo diagnóstico da alta hospitalar.

Por fim, para medir a taxa de eficiência de leito, foram propostas as análises dos seguintes dados: ${ }^{12}$

(1) Taxa de ocupação: cálculo da média de pacientes internados por dia e a capacidade operacional do hospital naquele dia. São considerados, inclusive os leitos de recuperação pós-anestésica, de observação, de berçário e pré-parto. Já os leitos bloqueados, por manutenção ou isolamento por doença, são excluídos;

(2) Média de permanência: relaciona o total de pacientes internados por dia em determinado período;

(3) Índice de renovação ou giro de rotatividade: Representa utilização do leito hospitalar durante o período considerado. Relaciona-se o número de saídas (altas e óbitos) / número de leitos no mesmo período. Importante para demostrar quantos pacientes um leito recebe no período. A eficiência no uso do leito é demonstrada com índices altos de renovação.

(4) Intervalo de substituição: consiste no tempo médio de desocupação de um leito entre a saída de um paciente e a admissão de outro. Para fazer esse cálculo, multiplica-se a média de permanência pelo percentual de desocupação e divide-se o resultado pela porcentagem de ocupação. Um índice alto interfere na assistência dos pacientes que esperam por um leito clínico ou cirúrgico.

Em seguida, os possíveis indicadores gerados com os dados públicos disponíveis, foram calculados e apresentados em tabelas e gráficos. Posteriormente, foram avaliados e validados pela equipe de pesquisadores formada por três profissionais: um gestor hospitalar, analista de dados, um coordenador de desospitalização e um cientista de dados.

\section{RESULTADOS E DISCUSSÕES}

Quanto aos indicadores para análise da desospitalização para o período de 2009 a 2019 foi possível identificar o total de 2.142 pacientes com transferências para outros estabelecimentos não especificando o tipo de instituição receptora ou serviço de saúde. Além disso, tem-se o número de 9.644 pacientes com permanência acima de 30 dias, porém não foi possível identificar o motivo de saída de cada um desses pacientes. 
O número de pacientes com reinternação em menos de 30 dias foi de 36.943 , já os que apresentaram o mesmo diagnóstico da alta hospitalar não estão descritos. Não foram alinhados na análise, os diagnósticos de entrada e saída das internações com as reinternações ocorridas. É importante salientar que os indicadores para medir a taxa de eficiência de leito, exprime informações importantes da necessidade ou não de redimensionamento ou adequação da estrutura de atendimento.

Com relação a taxa de ocupação, para a realização desse cálculo se fez necessário levantar o número de leitos disponíveis no período. O DATASUS informa apenas o número de leitos instalados, o que inclui leitos impossibilitados para uso (bloqueados para manutenção, limpeza, isolamento ou outro) e não os operacionais.

Sobre a média de permanência para cada $\mathrm{AlH}$ durante o período estudado foi de 6,3 dias, (variação entre 5,8 e 6,8 dias) sendo que são informadas internações de 1 a 400 dias de permanência. Já as internações com mais de 30 dias de permanência somam 9.644. Excluindo-se estes pacientes tem-se uma média de permanência de 4,6 dias (variação entre 4,5 e 4,8 dias) (Tabela 1).

Tabela 1. Média de permanência dos pacientes por ano analisado.

\begin{tabular}{|l|r|r|}
\hline Ano & $\begin{array}{l}\text { Média de permanência } \\
\text { (dias) }\end{array}$ & $\begin{array}{l}\text { Média para pacientes } \\
\text { com internação até 30 } \\
\text { dias (dias) }\end{array}$ \\
\hline 2009 & 6,1 & 4,5 \\
\hline 2010 & 5,9 & 4,5 \\
\hline 2011 & 6,2 & 4,5 \\
\hline 2012 & 6,6 & 4,6 \\
\hline 2013 & 6,8 & 4,8 \\
\hline 2014 & 6,4 & 4,6 \\
\hline 2015 & 6,4 & 4,5 \\
\hline 2016 & 6,5 & 4,5 \\
\hline 2017 & 6,7 & 4,7 \\
\hline 2018 & 6,3 & 4,5 \\
\hline 2019 & 5,8 & 4,6 \\
\hline Média & $\mathbf{6 , 3}$ & $\mathbf{4 , 6}$ \\
\hline
\end{tabular}

Com relação ao índice de renovação ou giro de rotatividade e do intervalo de substituição, os mesmos não puderam ser calculados como planejado devido a indisponibilidade do número de leitos operacionais da instituição no período analisado. De 2009 a 2019 o hospital teve 269.160 AlHs (Autorização para 
Internação Hospitalar) aprovadas para 174.717 pacientes em 232.217 internações e 36.943 reinternações (internação com período menor que 30 dias da última $\mathrm{AlH}$ ). Destes pacientes, 8.252 tiveram óbito na primeira internação, taxa de mortalidade $4,7 \%$. A taxa de mortalidade hospitalar foi de $5,3 \%$. O grupo "diagnóstico" com maior número de internações é o de doenças do sistema circulatório com 12,74\% (CID10 I00 a 199). Já para reinternações, a causa principal foram as neoplasias com 20,27\% (CID10 C00 a D48) (Tabela 2). Por fim na Tabela 3 são apresentados alguns indicadores gerais disponibilizados na análise de dados do período.

Tabela 2. Internações e reinternações por ano.

\begin{tabular}{|l|r|r|r|}
\hline Ano & Internações & Reinternações & $\begin{array}{l}\text { \% de } \\
\text { Reinternados }\end{array}$ \\
\hline 2009 & 28.968 & 3.844 & 13,7 \\
\hline 2010 & 29.904 & 4.506 & 15,5 \\
\hline 2011 & 25.304 & 3.612 & 14,7 \\
\hline 2012 & 25.899 & 3.477 & 13,9 \\
\hline 2013 & 24.602 & 3.061 & 12,9 \\
\hline 2014 & 26.095 & 3.281 & 13,0 \\
\hline 2015 & 24.025 & 3.375 & 14,5 \\
\hline 2016 & 22.269 & 2.907 & 13,5 \\
\hline 2017 & 18.365 & 2.449 & 13,7 \\
\hline 2018 & 20.099 & 2.583 & 13,4 \\
\hline 2019 & 23.720 & 4.021 & 17,4 \\
\hline Total & $\mathbf{2 3 2 . 2 1 7}$ & $\mathbf{3 6 . 9 4 3}$ & $\mathbf{1 4 , 1}$ \\
\hline
\end{tabular}

Tabela 3. Indicadores gerais.

\begin{tabular}{|l|r|}
\hline Leitos operacionais & Informações \\
\hline Leitos hospitalares & $\begin{array}{r}\text { Variação entre 766 e 1061 leitos, redução } \\
\text { de 14,2\% de 2009 a 2019 }\end{array}$ \\
\hline Paciente dia & Dado indisponível \\
\hline Internações & 269.160 \\
\hline $\begin{array}{l}\text { Reinternações em menos de } \\
30 \text { dias/ índice }\end{array}$ & $36.943,14,2 \%$ \\
\hline Altas & 250.844 \\
\hline
\end{tabular}




\begin{tabular}{|l|r|}
\hline Transferências externas & 2.142 \\
\hline $\begin{array}{l}\text { Total óbitos / Taxa de } \\
\text { mortalidade }\end{array}$ & $14.395 / 5,2 \%$ \\
\hline Outras saídas hospitalares & 6,3 \\
\hline $\begin{array}{l}\text { Média de permanência do } \\
\text { paciente }\end{array}$ & 4,6 dias / 9.644 \\
\hline $\begin{array}{l}\text { Média de permanência dos } \\
\text { pacientes excluindo os acima } \\
\text { de } 30 \text { dias (total) }\end{array}$ & 5,2 \\
\hline Média de diárias pagas & 4,2 \\
\hline $\begin{array}{l}\text { Média de diárias pagas dos } \\
\text { pacientes excluindo os acima } \\
\text { de } 30 \text { dias }\end{array}$ & Dado indisponível \\
\hline Taxa de rotatividade do leito & Dado indisponível \\
\hline Intervalo de substituição & 42 anos \\
\hline $\begin{array}{l}\text { Média de idade dos } \\
\text { pacientes }\end{array}$ & \\
\hline
\end{tabular}

Não foi possível analisar o percurso da jornada do paciente relacionado a transição do cuidado devido à dificuldade encontrada de não se ter acesso ao código de identificação do paciente no sistema para localizar seus atendimentos nos diversos serviços disponibilizados pelo hospital. Essa informação, inclusive, evidenciaria as estruturas disponíveis para transição de cuidados como a assistência domiciliar (homecare).

Outra dificuldade está na não disponibilidade de dados integrados entre os equipamentos de saúde para que se possa entender essa jornada do paciente. Ficou inviável saber se, após uma alta hospitalar, o paciente seguiu em um atendimento ambulatorial, serviço básico de saúde, pronto atendimento, ou mesmo, uma internação em outra instituição.

Neste trabalho foi criado um business key, que permitiu localizar as reinternações de pacientes com período menor que 30 dias da alta hospitalar. Além disso, tentou-se localizar as reinternações motivadas pela mesma doença (causa de entrada - CID 10), porém, isso é depende da hipótese diagnostica da equipe na reentrada. $O$ mais adequado seria ter um protocolo de internação onde fosse especificado na $\mathrm{AlH}$ a reinternação para continuidade ou revisão das condutas terapêuticas adotadas. A adequada análise das reinternações daria oportunidade para melhor avaliação da aderência do paciente ao tratamento, bem como da eficiência clínica da instituição com objetivo de implementação de ações para melhoria.

Por meio dos dados obtidos, observou-se que neste hospital houve uma redução de $14,2 \%$ no número de leitos no período estudado, mas não é 
possível saber quais os motivos e se houve aumento de leitos em outra instituição como contrapartida.

\section{CONSIDERAÇÕES FINAIS}

Para o paciente hospitalizado já com diagnóstico e tratamento, porém em recuperação prolongada, existem as seguintes possibilidades: unidades de transição ou retaguarda, unidades de cuidados paliativos e assistência domiciliar. Não há competição, mas sim complementação. Nos hospitais, o ideal seria o tratamento para o cuidado agudo de cada paciente. Após esse período, outras unidades de transição e retaguarda, somadas ao homecare, complementariam o cuidado, garantindo o atendimento adequado. Sem planejamento adequado da transição do cuidado ocorrem casos em que a alta é precoce. A expectativa é que o paciente termine o seu tratamento em casa ou procure a assistência ambulatorial. Mas a falta de controle e acompanhamento domiciliar na sequência pode acarretar o abandono do tratamento preconizado, gerando possíveis internações.

Atualmente, existem opções que fortalecem e fazem com que a coordenação do cuidado evolua. Com a grande rede de equipamentos de saúde disponíveis, a segurança do paciente deve ser sempre o centro de toda e qualquer decisão. As discussões sobre transição do cuidado são cada vez mais comuns, visando que o paciente deve ser tratado no lugar certo, no momento e tempo adequado. As diversas possibilidades do cuidado se completam e se conectam durante a jornada do paciente prevenindo agravamento dos casos e possibilitando mais segurança, especialidade na assistência, menor risco e custo para o serviço público de saúde.

No contexto hospitalar, o processo de desospitalização é um caminho que propõe a racionalização de custo e uma tendência no modelo da assistência aos pacientes complexos, que necessitam de apoio da equipe multiprofissional para poder retornar ao seu domicílio com segurança. É de fundamental importância a produção de novas formas de cuidar e de novas práticas de saúde com o compromisso de manter a excelência na assistência prestada.

\section{AGRADECIMENTOS}

Os autores agradecem a empresa Techtrials pela liberação e customização da ferramenta TT Hospital Explorer, em especial a Douglas Valverde e Élcio Firmiano.

\section{REFERÊNCIAS}


1. Datasus. Histórico do departamento de informática. Disponível em: https://datasus.saude.gov.br/sobre-o-datasus/

2. Ministério da saúde. Tutorial tabnet. Disponível em: http://tabnet.datasus.gov.br/Tutorial/Tutorial tabNet FINAL.pdf

3. Tabnet. Informações gerais. Disponível em: https://datasus.saude.gov.br/informacoes-de-saude-tabnet/

4. Tabnet. Capítulo 4 - fonte de informações. Disponível em: http://tabnet.datasus.gov.br/tabdata/livroidb/2ed/fontes.pdf

5. Netto AV, Tateyama AGP. Avaliação de tecnologia de telemonitoramento e biotelemetria para o cuidado híbrido para o idoso com condição crônica. Journal of Health Informatics. 2018; 10(4).

6. Valerio Netto, A. Quando a área de saúde encontra a ciência de dados. Disponível em: https://antoniovalerionetto.medium.com/quando-a$\%$ C3\%A1rea-de-sa\%C3\%BAde-encontra-a-ci\%C3\%AAncia-dos-dadosc6cf63007dda

7. Valerio Netto A, Petraroli AG. Modelagem de um sistema para o telemonitoramento de idosos com condição crônica baseado em biotelemetria. J. Health Inform. Jan.-Mar., 2020; 12(1):10-6.

8. Oliveira, LR. Descrição do serviço de desospitalização de um hospital privado no município de São Paulo, Dissertação de mestrado. São Paulo. Universidade Federal de São Paulo, 2018.

9. Anahp. Os desafios na gestão dos leitos de longa permanência. Revista Panorama ANAPH [internet]. 2015. Disponível em: https://issuu.com/anahp/docs/revpanorama 2015-mai jun-site

10. Mendes EV. As redes de atenção à saúde. Brasília: Organização PanAmericana da Saúde, p.549, 2011.

11. Polisaitis AEG. Cuidados pós-agudos: como estão inseridas nas políticas de saúde brasileira as Instituições de Longa Permanência para Idosos (ILPIs) e as clínicas de retaguarda? Disponível em:

http://bibliotecadigital.fgv.br/dspace/bitstream/handle/10438/24065/TA.Arian e.Polisaitis.VF.pdf

12. Zucchi P, Bittar OJ, Nogueira V, Haddad N. Produtividade em hospitais de ensino no estado de São Paulo, Brasil. Rev. Panam. Salud. Publica. Nov. 1998; 4(5). 
Recebido: 23 de março de 2021. Aceito: 31 de março de 2021

Correspondência: Antonio Valerio Netto. E-mail: avnetto@hotmail.com

Conflito de Interesses: os autores declararam não haver conflito de interesses.

(C) This is an Open Access article distributed under the terms of the Creative Commons Attribution License, which permits unrestricted use, distribution, and reproduction in any medium, provided the original work is properly cited 